\title{
FLASHES DE EMANCIPACIÓN EN LA ACTUACIÓN DOCENTE E INVESTIGACION UNIVERSITARIA DE LOS MIEMBROS DEL SISTEMA NACIONAL DE INVESTIGADORES (SNI)
}

\author{
FLASHES DA EMANCIPAÇÃO NA ATUAÇÃO DOCENTE E NA \\ PESQUISA UNIVERSITÁRIA DOS MEMBROS DO SISTEMA \\ NACIONAL DE PESQUISADORES
}

\section{FLASHES OF EMANCIPATION IN THE TEACHING ACTIVITY AND UNIVERSITY RESEARCH OF SNI MEMBERS}

\author{
Aristeo Santos Lopez ${ }^{1}$ \\ Universidad Autónoma del Estado de México (UAEMéx), Profesor investigador de la \\ Facultad de Ciencias de la Conducta
}

Resumen: El texto es una reflexión acerca del docente como un profesional que transita en lainvestigación; docencia; difusión y generación de la culturay del conocimiento. Para ello, se ha escuchado voces de algunos profesores que pertenecen al Sistema Nacional de Investigadores de México, adscritos en la Universidad Autónoma del Estado de México UAEMEX. Hallazgos: predominio de la investigación con enfoque positivista; falta de preparación y formación para la investigación; estudios desarrollados de manera individual dentro de sus soledades; poco apoyo de recursos materiales; sin derecho a descargas de tiempo; falta de espacios, así como de equidad para la divulgación de sus investigaciones; falta de incentivo económico; producción mecanicista; sentimiento de presiones y poco reconocimiento; pocas oportunidades para seguir con sus formaciones en instituciones dentro de los estándares de calidad educativa.

Palabras clave: Docencia. Investigación. Emancipación. Profesores. Universidad.

\footnotetext{
${ }^{1}$ Posdoctor por la Universidade de São Paulo, Faculdade de Economia, Administração e Contabilidade (FEA); Doctor en Educación (énfasis en Administración) por la Universidade Estadual de Campinas, São Paulo.
} 
Resumo: O objetivo deste texto é uma reflexão sobre o docente como um profissional que transita na pesquisa, na docência, difusão e geração da cultura e do conhecimento. Para isso, escutaram-se as vozes dos professores que pertencem ao Sistema Nacional de Pesquisadores de México, da Universidade Autônoma do Estado do México. Algumas conclusões: predomínio da pesquisa com enfoque positivista; falta de preparação e formação para a pesquisa; estudos realizados de maneira individual dentro da solidão de cada um dos pesquisadores; pouco apoio de recursos materias, assim como de tempo; falta de incentivo econômico; produção mecânica; sentimentos de pressões e pouco reconhecimento; faltam oportunidades para seguirem com as suas formações em instituições que estejam dentro de um padrão de alta qualidade educativa.

Palavras-chave: Docência. Pesquisa. Professores. Emancipação. Universidade.

Abstract: The text is a reflection on the teacher as a profesional who travels in Research; dissemination and generation of culture and knowledge. For that, we have heard voices of some teachers who belong to the National System of Researchers from Mexico, attached at the Autonomous University of Mexico state. Some of the findings: predominance of research positivist approach; lack of preparation and training for research; studies developed individually within their solitudes; little support to material resources; without the right to discharge of time; lack of space, as well as equity for the dissemination of their research; lack of economic incentive; mechanistic production; feeling of pressure and little recognition; few opportunities to continue their training in institutions within the standards of educational quality.

Keywords: Educational. Research. Teachers. Emancipation. University.

\section{PRIMER MOMENTO DEL DIÁLOGO}

La formación es un hacer permanente que se rehace constantemente en la acción.

Para poder ser, tiene que serse.

(Paulo Freire)

Lo que se puede percibir y afirmar es que el clima político e ideológico no parece favorable para los profesores en este momento. Sin embargo, el ritmo de incertidumbre, del vacío (LIPOVESTKY, 1986) dificultades, ética relativa, conocimiento volátil entre otras características permea todos los ambientes. En este marco y ya en 
escenarios de la educación superior, ellos tienen la oportunidad de comprometerse, haciendo la autocrítica respecto al ethos académico. Las posibilidades de ese encuentro si bien pueden ser amplias en momentos se destinan a la preparación, así como a los programas de perfeccionamiento del profesorado o las formas de colonización dominantes de la enseñanza en el aula.

Bajo esta tónica, los profesores y otras personas relacionadas a las instituciones educativas deben desarrollar una perspectiva teórica que redefina la naturaleza de la crisis educativa y al mismo tiempo les proporcione las bases para un punto de vista alternativo sobre su formación y trabajo. En síntesis, el reconocimiento de crisis educativa tiene mucho que ver con la tendencia progresiva a la reducción del papel de los profesores en todos los niveles educativos. Lentamente se ha impregnado la impotencia ante la suma de hechos que le van restando autonomía hasta de pensamiento. En esta idea, sería un prerrequisito teórico necesario para que los docentes se organicen con eficacia y dejen oír colectivamente sus voces. Además, este reconocimiento deberá luchar a brazo partido no sólo con la pérdida creciente de poder entre los profesores en lo que se refiere a las condiciones básicas de su trabajo, sino también contra una percepción pública sobre su papel como profesionales de la reflexión. Se suma a ello, la necesidad de defender a las instituciones educativas como instituciones esenciales para el mantenimiento y el desarrollo de una democracia crítica y para defender a los profesores como intelectuales transformadores que combinan la reflexión y la práctica académicas con el fin de educar a los estudiantes para que sean ciudadanos reflexivos y activos (GIROUX, 1988, p. 172).

Para percibir la importancia de la institución como un espacio para la reflexión y notar los cambios que ha ido sufriendo a lo largo del tiempo, es necesario retomar la cuestión del debate, del diálogo y de los intercambios de opinión entre las esferas públicas y privadas. Para algunos pragmáticos norteamericanos como Dewey (1977), la esfera pública es el nexo entre importantes ámbitos pedagógicos en los que la democracia, como movimiento social, está ligada al esfuerzo de numerosos grupos subordinados que tratan de acoger y producir un discurso social e identificar sus implicaciones para la acción política. Ampliando la opinión de Dewey, según la cual la acción social inteligente es portadora de las mejores promesas para una sociedad más humana, los re-construccionistas sociales de las décadas de 1930 y 1940 defendieron una política de individualidad social en la que los imperativos de la democracia se proponían como meta no sólo en las instituciones educativas, sino en todos los ámbitos pedagógicos que reconocían la primacía de la política en la vida cotidiana. 
Bajo la lógica menciona se escondía un énfasis en la relación entre el conocimiento y el poder, la conducta y la simulación y el compromiso y la lucha colectiva. De hecho, la esfera pública no sólo servía para producir el lenguaje de la libertad, sino que además mantenía viva la esperanza de los grupos subordinados en tanto que podrían producir, algún día, sus propios intelectuales. En las palabras de Gramsci (1997), esto significa la creación de intelectuales orgánicos, los que podrían, por una parte, llenar el vacío existente entre las instituciones académicas y los temas específicos, y, por otra, las labores de cada día. Es decir, esos intelectuales podrían proporcionar las habilidades morales y políticas necesarias para consolidar instituciones de educación popular y culturas y creencias alternativas.

De esta manera queda planteado que las instituciones educativas, principalmente aquellas para la educación de los profesores, necesitan un replanteamiento como esferas públicas. Según Giroux (1988), las instituciones tal como hoy existen están peligrosamente desprovistas tanto de conciencia como de concientización social. Consiguientemente, urge desarrollar programas que posibiliten que los futuros profesores sean educados como intelectuales transformadores capaces de defender y practicar el discurso de la libertad y la democracia. ${ }^{22}$

La educación del profesor raramente ha ocupado un espacio crítico, público o político dentro de la cultura contemporánea. En el campo de lo formal pareciera ser que este espíritu crítico debe ser proporcionado por los posgrados y no desde su biografía formativa. No hay lugar para que el significado de lo social pueda ser recuperado y reafirmado de forma que las historias culturales de profesores y estudiantes, sus narraciones personales y voluntad colectiva puedan aglutinarse en torno al desarrollo de una esfera contrapública democrática. De hecho, es razonable pensar que los programas para la formación de los profesores están diseñados para crear intelectuales que actúen al servicio de los intereses del estado y cuya función social primaria se centra en el mantenimiento y la legitimación del status quo. Esto, según Puiggrós (1990), se puede percibir claramente en México, donde el sujeto pedagógico moderno $^{33}$ puede reproducirse en un sistema educativo complejo, con un discurso escolar estructurado por un partido que por más de sesenta años fue el gobernante y ocupa un gran espacio en el sistema educativo. Tomando cuenta de todas las estructuras y

\footnotetext{
${ }^{2}$ La educación pretende ser un proceso de transmisión unilateral en el proyecto educativo moderno diseñado por las pedagogías dominantes durante los últimos siglos. Pero podría ser de otra manera: Gramsci y Freire otorgan al vínculo pedagógico la posibilidad de realizar el proceso de reproducción-transformacióncreación de la cultura. Ese postulado pone en crisis al sujeto pedagógico moderno, pues una de las fuentes de su legitimidad es su autoidentificación con la definición de educación (PUIGGRÓS, 1990, p. 45).

${ }^{3}$ Por sujeto pedagógico moderno, según Puiggrós, se entiende al mediador entre los sujetos políticos y sociales actuantes en la sociedad y el "habitus" que se pretende inculcar (PUIGGRÓS, 1990, p. 41).
} 
pensamientos inundando no solo a las instituciones sino el quehacer y cotidiano de los participantes.

Al contemplar a los profesores como transformadores se estaría considerando que:

Toda actividad humana implica alguna forma de pensamiento. Así se exalta la capacidad humana de integrar pensamiento y praxis, ${ }^{44}$ resaltando la importancia del profesor como profesional reflexivo de la enseñanza;

a) Esta visión es una fuerte crítica a las ideologías tecnocráticas e instrumentales subyacentes en una teoría educativa que separa la conceptualización, la planificación y el diseño de la currícula de los procesos de aplicación y ejecución; y

b) Contribuye a aclarar el papel que desempeñan los profesores en la producción y legitimación del estado, mencionado en el párrafo anterior.

c) Orientar las funciones y roles del profesor hacia su compromiso con su comunidad; hacia las necesidades sociales, de supervivencia, de mejoría de vida, de desarrollo humano. Un sujeto que rompa las esferas del salón de clase para sumarse a proyectos que conduzcan su comunidad a solución de problemas colectivos. Uno de los autores que nos presente profundas reflexiones, en muchas de sus obras, acerca de éste tema es Santos (1989, 1997, 2004, 2008).

Siendo así, al considerar a los profesores como sujetos transformadores, también es importante resaltar, en términos políticos y normativos, las funciones sociales concretas que realizan los docentes. De esta manera es necesario mirar a las instituciones educativas como lugares económicos, culturales y sociales ligados inseparablemente a los temas del poder y el control. Así las instituciones educativas no se limitarían a transmitir un conjunto de conocimientos y valores, sino que serían lugares que representan formas de conocimiento, usos lingüísticos, relaciones sociales y valores que implican selecciones y exclusiones particulares a partir de la cultura general.

Según Giroux (1988), de esta forma las instituciones educativas servirían para introducir y legitimar formas particulares de vida social. Más que instituciones

\footnotetext{
${ }^{4}$ Aquí se entiende praxis de acuerdo al pensamiento de Agnes Heller, quien la define como acciones conscientes (HELLER, 1982a, p. 20).
} 
objetivas alejadas de la dinámica de la política y el poder, las escuelas son esferas debatidas que encarnan y expresan una cierta lucha sobre qué formas de autoridad, tipos de conocimiento, regulación moral e interpretaciones del pasado y del futuro deben ser legitimadas y transmitidas a los estudiantes. Las instituciones no son espacios neutrales y, consiguientemente, tampoco los profesores pueden adoptar una postura neutral. En el sentido más amplio, los profesores, han de contemplarse en función de los intereses ideológicos y políticos que estructuran la naturaleza del discurso, las relaciones sociales del aula y los valores que ellos mismos legitiman en su enseñanza.

Entonces, si los profesores han de educar a los estudiantes para ser ciudadanos activos y críticos, deberían convertirse ellos mismos en intelectuales transformadores para generar un conocimiento como emancipación y no un conocimiento como regulación. Según Puiggrós (1990), la democratización del sujeto pedagógico es indispensable si se pretende desarrollar un sistema complejo que proporcione múltiples opciones sin que esto implique aumentar la polarización cultural ni concurrir a la reproducción de la creciente desigualdad social.

Giroux (1988), al igual que Freire (1974), piensa que uno de los componentes centrales de la categoría de los intelectuales transformadores es la necesidad de conseguir que lo pedagógico sea más político y lo político más pedagógico. Hacer lo pedagógico más político significa insertar la instrucción escolar directamente dentro de la esfera política al demostrar que dicha instrucción representa una lucha para determinar el significado y, al mismo tiempo, una lucha en torno a las relaciones de poder. Dentro de esta perspectiva, la reflexión y la acción críticas se convierten en parte de un proyecto social fundamental para ayudar a los estudiantes a desarrollar una fe duradera en la lucha contra las injusticias económicas, políticas y sociales, así como para humanizarse más como parte de esa lucha.

Hacer lo político más pedagógico significa servirse de la pedagogía para encarnar intereses políticos de naturaleza liberadora. Es servirse de formas pedagógicas que traten a los estudiantes como sujetos críticos, hacer problemático el conocimiento, recurrir al diálogo crítico y afirmativo y llegar a consensos. Por lo tanto, el punto de partida pedagógico para este tipo de intelectuales no es el estudiante aislado, sino los individuos en grupos dentro de sus múltiples contextos culturales, sociales, raciales, históricos, sexuales, mirados conjuntamente con la particularidad de sus intereses, problemas, sueños, aspiraciones y desencantos. Como dice Heller (1982b), una relación libertadora es aquella en la que el otro deja de ser objeto y pasa a ser objetivo.

En esta idea para construir la formación emancipadora, una de las funciones más importantes a desempeñar por el profesor sería la de poner en duda los 
principios, las ideas, las interpretaciones, para que fuese el propio alumno el que eligiera las que le conviniesen o se acoplasen más a su forma de ser, a su forma de vida, a su idiosincrasia, implicando la capacidad de pensar libremente y de buscar nuevos conocimientos. En este sentido, se pueden distinguir dos tipos de educación: una que trata exclusivamente de que el alumno aprenda la verdad ya dada y, otra, que sería emprender juntos un camino de preguntas, de dudas y de respuestas parciales y transitorias, para ir construyendo cada uno su propia existencia. Para ello, el profesor tiene que reconstruirse constantemente en los ejes de la docencia, de la investigación, como generador de cultura y difusor de la misma, así como de los conocimientos. Sin embargo, es oportuno señalar que la posibilidad de formar sujetos emancipados siempre ha existido y siempre ha sido relativa, puesto que las posibilidades y las limitaciones de liberación han estado presentes de forma permanente.

Se puede decir que la complejidad para formar sujetos críticos y reflexivos estriba en algunos puntos como: a) en el rescate de los valores fundamentales, lo cual dependería en gran parte de la participación y compromiso de los profesores; b) en el hecho de que el sentido del profesor universitario se diluye porque no puede ser modelo, ni dar testimonio para transmitir valores encaminados al ejercicio de la libertad cuando él mismo está inmerso en la lucha por su supervivencia básica, ya que tiene que buscar una pluralidad de alternativas para satisfacer sus necesidades elementales; c) la necesidad, entonces, de una transformación primero a nivel individual donde el profesor gire hacia sí mismo para construirse como un ser emancipado y, posteriormente, hacia los demás a través de sus prácticas, para generar ambientes críticos y realizar una labor que lo conduzca su propia dignificación a través del ejercicio de su vocación; d) la generación colectiva de cambios en todo el sistema educativo a través de la construcción de proyectos de nación, estatales y locales; e) en lo que se refiere a muchas universidades en América Latina es necesaria una reforma estructural con claridad y solidez que permitan la construcción de sus propias identidades.

En esta perspectiva, se podría creer en la innovación en las instituciones educativas: cuando el propio conocimiento pasa a ser cuestionado, cuando se analizan sus medios y fines, cuando el conocimiento puede llegar a la crítica y a una transformación individual y colectiva de la sociedad. El deseo y la necesidad de que ocurran reformas que conduzcan, no sólo la universidad sino a todo el sistema educativo, a un cambio estructural, administrativo y pedagógico, son explícitas en todos los discursos como eje central para resignificar valores, orientar la misión y los fines de la educación, rescatar la parte humana en la formación del ser, y aproximar a la universidad y a la educación a la sociedad y sus demandas. En ese reclamo a los cambios, a una 
renovación, podríamos indagar acerca de otro discurso que está presente en varios y diferentes segmentos, incluido el mundo académico: la innovación.

La innovación es interpretada de acuerdo a diferentes conceptos, tales como:

a) Desde el punto de vista lingüístico. La innovación sería lo nuevo, lo que no existía previamente. En la cuestión teórica resalta el hecho de que las sociedades siempre han tratado de sintetizar lo nuevo dentro de la tradición, porque ambas dimensiones son importantes para el ser humano: sin la tradición y la historia él no sería nada y sin acceder a lo nuevo quedaría fuera de la realidad global. Se supone que todo el conocimiento científico es innovador, porque trata de añadir algo nuevo a lo que ya existe. Otra cuestión es la forma en que ese conocimiento se debe transmitir y si la innovación es profunda o superficial;

b) En términos de pensamiento y actitudes, la innovación sería algo que permanece y que, a diferencia de la moda, se vuelve clásico al cambiar a los demás y a la realidad por completo $\mathrm{y}$, en consecuencia, al paso del tiempo, sigue teniendo vigencia. Se considera innovador aquel que trae algo que permita tener una visión distinta del mundo, y para eso es necesario ser capaces de difundir, de atreverse, de hacer, de no quedarse con la intención de desarrollar una investigación, de emprender la búsqueda (primordialmente en equipos donde se estudie y se trabaje con una participación plural que permita el desarrollo de las habilidades de todos, lo que exige la disposición y tendencia personal). Este desarrollo de habilidades no implicaría simplemente acumular información, sino establecer relaciones de la misma con el entorno, de tal forma que se busquen nuevos caminos que ayuden a la sociedad;

c) bajo el enfoque filosófico, la innovación es interpretada como una mejor formulación de las preguntas sobre las consecuencias de lo que estamos haciendo: preguntar más que afirmar. Innovación entonces sería la búsqueda incesante de nuevas explicaciones a fenómenos viejos o a fenómenos nuevos, teniendo como norte la justicia más que la verdad, dedicando más esfuerzos a construir una sociedad mejor que a encontrar la verdad última de las cosas. En esta búsqueda de explicaciones entraría la posibilidad de reflexionar, de repensar, seguramente con base en un pretexto 
y un contexto, el texto que se está pensando, pues al ser repensado se permitirá vislumbrar una nueva interpretación para una realidad actual;

d) En relación con la pedagogía la innovación se observaría en aquellas pedagogías que conduzcan al alumno a desarrollar sus propios procesos de pensamiento, a reflexionar, a hacer correlaciones y a construir el conocimiento. Dentro de esta perspectiva la tecnología interviene solamente como una herramienta;

e) En lo tecnológico, la innovación sería descubrir algo nuevo, respecto a lo que ya existe, que permita lograr avances en el sentido de reducir costos y aumentar la rapidez.

La innovación es interpretada casi de manera unánime de forma diferente a la interpretación funcionalista y neoliberal. Sin embargo, se reconoce que actualmente son estos paradigmas los que imperan en las universidades.

Los intelectuales transformadores necesitan desarrollar teorías que conduzcan a la praxis y que en realidad posibiliten cambios. Indiscutiblemente los intelectuales transformadores tienen que comprometerse y manifestarse contra las grandes injusticias, tanto dentro de las instituciones como fuera de las mismas. Ellos tienen que crear, a través de sus pensamientos y acciones, las condiciones para que sus estudiantes, mediante los conocimientos y valores desarrollados en este contexto, se vuelvan intelectuales en el sentido en que Gramsci y Freire los describen. ${ }^{55}$

La realidad planteada por las contradicciones del proyecto de la modernidad, entre otras cosas, impactó al mundo, condujo al hombre a la desesperanza, a la falta de indignación, a las desigualdades, a la violencia y al dominio del hombre sobre la naturaleza. Adorno ya decía que después de Auschwitz la educación jamás sería la misma. La exigencia de que Auschwitz no se repita es la primera de todas en la educación, pues ese hecho fue la barbarie contra la cual se dirige toda educación. Actualmente tenemos otros hechos que impactan al mundo y que vienen a sumarse a los eventos de las primera y segunda guerras mundial del siglo pasado.

Vemos países europeos en la más absoluta crisis económica; asistimos los conflictos de discriminación, intolerancia e barbarie desatados por pueblos orientales

\footnotetext{
${ }^{5}$ Ambos intentan criticar explícitamente el concepto actual de educación y superar el vínculo pedagógico que caracteriza no solamente las relaciones escolares, sino aquellas de índole político-pedagógica que recorren toda trama social. Ambos cuestionan el papel de los intelectuales en la construcción de la cultura dentro de la sociedad moderna. En los dos, el vínculo pedagógico es también político y se define como conflicto y no como espacio neutral y renuente a la lucha.
} 
que dan sus interpretaciones propias bajo sus creencias; la pobreza que aumenta no solo en África como en el mundo; Rusia y Ucrania a punto de desatar una guerra movida no solo por medir el poder sino por la presión de la industria bélica; vivimos en países de América Latina como Brasil y México que la corrupción y la violencia están estampadas en el cotidiano; que hablar de Perú y de Bolivia y la marginación de sus indígenas; Colombia y ahora también México conocidos por el narcotráfico. Finalmente, hoy por hoy, el discurso y las vivencias que nos indignan, que nos asombren, que nos mueven, huyen del eje de Auschwitz, del eurocentrismo e se instalan por todo el mundo con distintas matices que hieren los derechos humanos de los ciudadanos.

En este sentido, lo que urge es lo que Adorno (1993), llama el "giro" hacia el sujeto. Se deben descubrir los mecanismos que vuelven a los hombres capaces de tales atrocidades y mostrárselos a ellos mismos para tratar de impedir que vuelvan a ser así, al mismo tiempo que se despierta una conciencia general respecto de tales mecanismos. Es la insensibilidad la que hay que combatir. Es necesario disuadir a los hombres de golpear hacia el exterior sin reflexionar sobre sí mismos. La educación carecería absolutamente de sentido si no fuese educación para una autorreflexión crítica.

Es el hombre quien hace su propia historia. La historia humana no es, por mucho, una historia natural. El hombre no se humaniza para cumplir los dictámenes de la naturaleza. De acuerdo a este pensamiento, se podría postular que la revolución para la transformación pedagógica y para la transformación del ser, primeramente pasa por la subjetividad, por la participación en un proceso lento y celular, por una revolución invisible en la cual alumnos y profesores pasan de la particularidad hacia la individualidad y hacia el propio proceso revolucionario. $Y$ es en este momento cuando el hombre toma conciencia de su alienación, de su realidad, de sus sentimientos, de su dureza, de su capacidad destructora, de su explotación y de su condición humana. Todavía no se sabe lo que se quiere o lo que sea lo mejor para la educación, pero sí se sabe lo que no se quiere repetir.

También se puede decir que se sabe que el hombre del futuro ya existe; ahora lo que se pregunta es: ¿qué tipo de hombre se quiere formar? También se sabe que el profesor puede ser un sujeto transformador, pero aún se buscan los cambios epistemológico-pedagógico-político-ideológico-cultural necesarios para que eso suceda, para que el profesor se involucre en proyectos que estén directamente relacionados con las necesidades de la sociedad y se establezca así una relación dialógica entre la sociedad y la universidad.

Del mismo modo, también ya fue constatado que la innovación en el plano cognitivo-instrumental sirve para la perpetuación del conocimiento como regulación, 
por lo tanto lo que se propone es que la innovación sea considerada como un instrumento para la negación de la regulación, que sea un instrumento que genere nuevas relaciones entre el ser, el saber y la acción, un cambio en los paradigmas de poder.

A seguir, se mencionará la opinión, la voz de algunos profesores de la Universidad Autónoma del Estado de México quienes están involucrados activamente en esta tarea de resignificar la docencia universitaria, pues hacen parte del Sistema Nacional de Investigación, lo que supone ser sujetos que ejercen la crítica y que buscan crear el conocimiento, preservar y generar la cultura, así como orientar el esfuerzo en la extensión de los mismos hacia la sociedad. Por ello, los ejes reflexionados fueron la propia crisis de la universidad; la función de investigación; la docencia; los valores que permean la labor en la universidad; la movilidad social; la vinculación entre universidad y sociedad.

\section{EL SEGUNDO MOMENTO DEL DIÁLOGO}

La propuesta fundamental de esta investigación, además de ser una reflexión sobre la universidad, su trayectoria y sus valores, es escuchar las voces de los académicos investigadores y sus opiniones y sentimientos sobre la universidad en que se encuentran insertos. Para ello, se buscó a cada uno de los investigadores de la Universidad Autónoma del Estado de México, quienes pertenecen al Sistema nacional de Investigadores, para invitarles a conceder una entrevista semi estructurada. Dicha entrevista fue construida a partir de las siguientes categorías: visión (percepciones de la relación de la ciencia, sociedad y universidad); movilidad social; tecnología; innovación; valores (cuáles permean las funciones de la universidad); sujeto emancipador. El estudio llevó dos años entre la revisión teórica, aplicación e interpretación de las entrevistas a 27 doctores que permitieron participar del estudio y, la conclusión de los análisis de los discursos y prácticas. En lo que sigue se buscará transmitir la interpretación de sus voces de manera global, con el objetivo de aproximarnos lo más posible a la realidad de sus pensamientos.

En lo que se refiere a la visión global de la universidad, de la ciencia y de la sociedad, se encontraron algunos discursos como la predominancia del enfoque positivista, hecho que no permite la transformación de la universidad para que deje de ser técnica y logre ser vocacional. Del mismo modo parece que la universidad está en un momento de caos, ya que al no existir un proyecto de nación, existen muchas leyes mas no un Estado de derecho y frente a esto el modelo se empalma con el modelo educativo si es que lo hay y no es posible construir un proyecto de universidad 
y mucho menos desarrollar una vinculación adecuada con la sociedad. Cómo se va a canalizar el quehacer científico de la universidad, si no se han resuelto discusiones básicas en torno a la misma, tales como: si es privada o pública, discusión que está desviando la profunda reflexión acerca de la misión de la universidad, incluyendo su vinculación con la sociedad.

Bajo la interpretación de que la universidad debería ser la conciencia de la sociedad, la primera estaría en crisis debido a que en algún momento parece que la sociedad se desconectó de su conciencia. Es una idea que se torna un hecho concreto, al observar la diferencia entre los discursos al interior de la universidad y la realidad imperante en la sociedad y aun dentro de la propia universidad. Dicha fragmentación entre teoría y praxis muestran un comportamiento esquizofrénico cuando se presencian actos que reflejan alineación, mecanización, catarsis, etcétera; en contraposición, existen discursos que apelan a la vinculación del ser con su propia conciencia y responsabilidad. Hoy por hoy, la universidad no está cumpliendo con su objetivo, sin embargo, dentro de este caos, se puede construir un orden y construir proyectos que nos den referentes éticos para encontrar una razón de ser.

Con relación a esta crisis, uno de los grandes problemas del sistema educativo, no sólo de México sino prácticamente de todo el mundo, consiste en que hay un divorcio marcado, no total, no insalvable, pero sí un divorcio marcado entre los sistemas educativos y la realidad de lo cotidiano, en el que se mueven, en el que se van a enfrentar las personas, entonces, sí hay un divorcio que convendría acortar o disminuir obviamente, que lo que se enseñase tuviese mucho más que ver con la vida y con la vida no económica solamente, sino con la vida de la persona en integridad más o menos global...buena parte de lo que se enseña en las universidades y en el sistema educativo en general, tienen que ver fundamentalmente con la reproducción del propio sistema.

Los profesores reconocen que no sólo la universidad, sino todo el sistema educativo, atraviesa por una divergencia marcada entre lo que se enseña y la realidad de lo cotidiano. Tal parece que el sistema educativo se limita a reproducir al propio sistema en lugar de reaccionar y promover cambios que generen lazos con los aspectos externos, no sólo con los económicos, sino también con cuestiones filosóficas e históricas:

De esta forma, como reproductora del propio sistema y manteniendo el modelo tradicional, en el que el profesor expone y el alumno es receptor, la universidad se está rezagando. Aunado al hecho de que la universidad no llega a la sociedad, es decir, no se aproxima, no comparte, ni difunde. A través del vínculo universidad 
y sociedad es que surge la universidad social, que bajo este término debería otorgar los servicios que la sociedad demanda, tales como hospitales, atención psicológica y social, e incluso promoción del arte, de tal forma que la universidad intente rescatar a la sociedad del torbellino de información comercial en que se encuentra inmersa.

En la misma línea, la universidad debería ser un punto de generación del conocimiento universal y restablecer los vínculos necesarios para que dicho conocimiento sea devuelto a la sociedad tal cual y se obtenga un beneficio concreto. Por otra parte, la realidad refleja que las universidades se han masificado y no garantizan la pureza del conocimiento, ni la permanencia del mismo, tampoco la preparación adecuada del egresado que lo coloque en condiciones de poner en práctica lo aprendido dentro de la sociedad, corriendo el riesgo de que la ciencia se vuelva un dogma.

Sobre la estructura de las universidades en Latinoamérica, los profesores colocan que las universidades presentan un problema estructural y piramidal. Paralelamente, las universidades crecen a un ritmo tan acelerado, que no permiten la asimilación de nuevos aprendizajes. En términos generales, requieren de una reforma estructural de fondo desde la elección de los responsables de la administración, los cambios de la misma, hasta la política educativa que se traza en sus interiores.

Ésa reforma tendría que contemplar además, la conformación de una nueva orientación que desplace la administración centralizada, la corrupción, los privilegios y el clientelismo, así como el predominio del "político sobre el académico". Dicha reforma debería contemplar el desempeño efectivo del consejo académico, de tal forma que esté en condiciones de sustentar la continuidad de los proyectos importantes, independientemente de los cambios de administración. Una reforma estructural en la que las autoridades administrativas, tanto al nivel de rectoría como de dirección de facultades, tuviesen un perfil más académico y no solamente político.

Dentro de esa visión de universidad, los profesores también expresaron sus pensamientos sobre la investigación, la docencia y los obstáculos a los que se enfrentan en estas actividades, así como los desafíos que les impone su cotidianeidad. Los investigadores reconocen que, a pesar de que la investigación no pertenece exclusivamente al espacio de la universidad, la universidad es todavía uno de los pocos sitios en México donde se puede realizar investigación.

Otra interpretación importante es que los investigadores reconocen que la investigación y la docencia no deberían estar aisladas, ya que la unión de ambas hace que se enriquezca la formación de los alumnos e incluso puede llegar a despertarles el interés por la investigación: La ventaja de que el investigador actúe como docente permite la transmisión de conocimientos de punta a las nuevas generaciones. 
Del mismo modo, es consenso general entre los profesores, que para hacer ciencia solamente se requiere de la disposición de pensar, ejercicio que debería ser desarrollado dentro del sistema educativo. Agregan, en lo que se refiere al ambiente, que para que esto sea posible las facultades deberían contar con grupos menores donde la dinámica profesor-alumno permitiera crear relaciones más humanas, de mayor compañerismo, de ayuda mutua y de trabajo en equipo.

El profesor asume que tiene la gran responsabilidad de crear el conocimiento; evaluar cuidadosamente sus beneficios para la sociedad; vincular ambos aspectos adecuadamente y difundir el conocimiento de tal forma que los prejuicios de interpretación de la sociedad no se conviertan en obstáculos para su aceptación y aplicación. Además, aseguran que se debería mejorar el vínculo con la sociedad al promover una comunicación interactiva; de esta forma, la sociedad expresaría sus necesidades al sector de investigadores y ellos podrían atenderlas oportunamente. De la misma manera en que están conscientes de sus responsabilidades, los profesores no desconocen los obstáculos y dificultades ante los que se enfrentan en la cotidianeidad para desempeñar la función de investigación. A continuación, se mencionan algunos obstáculos y dificultades:

1. De forma general, la falta, los vacíos en políticas educacionales de reglamentos referentes a la investigación, tales como:

a) En la UAEM, no existe la función del investigador; tampoco de la categoría;

b) La ausencia de considerar la posibilidad de acceso del investigador a otros espacios geográficos;

c) La insuficiencia de intercambio de investigadores y de cooperación en proyectos, en los que exista una definición clara de los criterios que normarían los mismos. La ausencia de una normatividad en investigación ocasiona que los pocos intercambios o co-participaciones en proyectos se lleven a cabo por mecanismos de instituciones externas a la universidad o por iniciativas personales;

d) La inexistente factibilidad de trabajos inter y multidisciplinarios;

e) La falta de criterios para tamizar los proyectos propuestos que deberían ser prioritarios; 
2. Carencia de una infraestructura adecuada para que el científico pueda dedicar parte de su tiempo a atender problemas de demanda social y el resto a problemas vinculados con la industria.

3. Necesidad de implementar la formación de grandes institutos con cuerpos colectivos de trabajo de investigación que sustituyesen la tendencia individualista;

4. Pocos estudios orientados a las demandas regionales. El trabajo de investigación debería estar direccionado más hacia lo regional que a lo nacional o internacional con el objetivo de responder a las demandas reales del entorno y así fomentar una amplia difusión local del trabajo de investigación generado;

5. Falta de experiencia y de iniciativa personal para enfrentar ciertos retos dentro de la investigación;

6. Puesto que toda su formación académica careció de elementos que le permitiesen desarrollar la investigación, el docente se ve en el verdadero reto de convertirse en investigador;

7. El hecho de que la investigación es incipiente en muchas universidades;

8. Los obstáculos generados por el manejo burocrático y discrecional de los recursos;

9. De igual forma, la existencia del favoritismo y el manejo de poder;

10. La poca disposición de los investigadores para aceptar la crítica y las diferencias de opinión;

11. La discriminación por género y el malestar de aquellos docentes de mayor antigüedad que no han alcanzado títulos de posgrado;

12. El poco intercambio con los mismos investigadores y la ausencia de un clima académico;

13. La falta de herramientas de trabajo;

14. Hay demasiada presión para producir y generalmente no importa la calidad o el alcance de lo que se produce. Las evaluaciones practicadas al interior del Sistema Nacional de Investigación tienen un matiz cuantitativo más que cualitativo;

15. Las fuertes cargas de trabajo y la diversidad de funciones impiden la concentración para la búsqueda de soluciones a los problemas que la sociedad demanda. Del mismo modo, se ve afectado el flujo armónico del tejido social porque no se observa cabalmente una división del trabajo. Los trabajadores e investigadores tienen la sensación de ser explotados, pues en necesario que desarrollen funciones disímbolas de las propias. Todo esto provoca que el ejercicio profesional se degrade y, por lo tanto, se degrade la propia dignidad personal, la cual está relacionada con el quehacer particular de cada quién dentro de la universidad. Se debe pensar en plantear un cambio y éste requiere, de manera indispensable, un reorde- 
namiento de los factores expuestos para devolver, en primer término, la dignidad a cada universitario - cualesquiera que sean sus funciones- de tal manera que se permita reintegrar en el interior de la institución el respeto en todas direcciones. Sólo desde un espacio de dignidad personal, respeto y principios éticos se tendría el sostén para el vínculo con la sociedad.

Con relación a la docencia y al ambiente en general, se pudo interpretar que los profesores perciben:

1. Desinterés y desinformación; los alumnos llegan a las licenciaturas sin capital cultural, sin lecturas, sin capacidad de análisis, sin saber escribir.

2. Desmotivación en los alumnos; alumnos pasivos; sin preguntas; sin curiosidad;

3. Alumnos con muchas carencias emocionales, con necesidades de afectos, de ser escuchados. Antes que generar climas de aprendizajes, el profesor tiene que generar espacios de escucha, de catarsis, de apapachos, de terapia;

4. Quizás por la apertura de actitudes y de comportamientos y de derechos humanos que se han conquistados, los alumnos presentan en los salones de clase estado de embriaguez, de uso de drogas, de salidas y entradas recurrentes; de frecuentes salidas de los salones ocasionadas por mal estar súbitos: falta de alimentos; ataques de histerismos; epilepsia, ataques de asma, mareos constantes. Todo ello, impacta el clima de aprendizaje y de convivencia, pues incluso los colegas pasan a tener miedos, se sienten presionados y estresados con lo que pueden ocurrir.

5. Falta de tolerancia para convivir con la diversidad humana, tanto por parte de los alumnos como de los profesores: creencias; género, raza; condición económica; discapacidades, entre otros.

6. Ausencia de ambientes que eduquen, que formen para la sensibilidad: alumnos, profesores, directivos circulan en su cotidianidad sin la sensibilidad desarrollada para colocarse en el lugar del otro.

7. Ambiente superficial y sin sentido.

8. Inconformidad de los profesores y actitud de reproche acompañada de pasividad;

9. Espíritu de supervivencia: hay que mantener la facultad para subsistir;

10. Falta de flexibilidad y agilidad en la actualización de programas y contenidos curriculares para responder a la velocidad de cambios característicos de nuestro tiempo;

11. La falta de personas de tiempo completo, investigadores y personas comprometidas y creativas; 
12. Necesidad de actualización constante, prioritariamente en el renglón de sistemas de comunicación;

13. Falta de compromiso de los profesores de asignatura;

14. Falta de compromiso, de forma general, para expresar aquello que se requiere cambiar en la institución, ya sea por comodidad o por temor a represalias;

15. La investigación en la universidad se ha generado una especie de esquema de clases sociales en el cual los investigadores se posicionan en un nivel superior a los docentes y esto da lugar a una competencia desleal. Hay una fragmentación entre investigación y docencia, de igual forma entre los sujetos.

16. La baja remuneración que conduce a los profesores a asumir muchas aulas en distintas instituciones educativas para obtener su supervivencia. De igual modo, ello repercute en la falta de tiempo para planear sus clases. La carencia económica también es un obstáculo para invertir en sus capacitaciones, en participar de congresos, en comprar las bibliografías adecuadas y necesarias, así como el acceso a la tecnología.

De las reflexiones anteriores sobre la visión global de la universidad y, en particular, de las dificultades y obstáculos dentro de la investigación y la docencia, se dio paso al cuestionamiento siguiente: ¿Continúa siendo la universidad un símbolo de movilidad social? Las respuestas fueron de tal inventiva que generaron una categoría adicional.

Acerca de la movilidad social, los discursos reflejan diferentes posturas que van desde quienes deslindan a la universidad de toda responsabilidad, hasta quienes afirman que la universidad no está cumpliendo con su objetivo de formar profesionales para el mercado laboral. Las posturas de los profesores son que la universidad no es responsable por la movilidad social. En la afirmación anterior los argumentos presentados afirman que la garantía de un empleo por parte del egresado depende de su formación e interés personal. La responsabilidad de la universidad se limita formar a los alumnos lo mejor posible, con los temas más actuales, y a motivarlos a continuar con estudios de posgrado, pues así, la perspectiva de conseguir un empleo podría ampliarse. La licenciatura debería proporcionar las bases para que cada egresado organizara su propio perfil, pero, en esta perspectiva, la responsabilidad de conquistar la movilidad social queda en el compromiso personal del egresado y en su desempeño.

Junto a las posturas acerca de la movilidad social se percibe que, para diferentes segmentos - tanto fuera como dentro de la universidad-, es un hecho que la universidad ya no es garantía de movilidad social como lo era en otros tiempos. Con- 
secuentemente, se genera una ausencia de legitimidad social y se rompen o deterioran las relaciones de autoridad moral. Un egresado de la universidad no goza más de la autoridad moral que se le confería a cualquier profesionista universitario.

Las generaciones actuales nacen en este ambiente caótico, en el que no tienen proyectos alternativos ofrecidos por los adultos, ni herramientas para construir sus propios proyectos. Sin embargo, los jóvenes de estas generaciones podrían ubicarse en dos posturas: la primera son aquellos que están en la búsqueda de sus propios referentes éticos; y la segunda, es un conjunto de jóvenes que cada vez se individualiza más. Y, dentro de esta idea de individualización, se percibe que la universidad pública, cuya misión debería ser formar entes de compromiso social, se está dejando invadir por la inercia de los discursos de la universidad privada, que cada vez con más fuerza promueve la formación de individuos competitivos que buscan un beneficio particular, esto conducido por el neoliberalismo y la globalización. De tal forma, que la universidad pública ha derrumbado los antiguos referentes éticos que de alguna manera servían de base para la reflexión sobre el hombre.

En el no cumplimiento de sus funciones, la universidad está invadida por nuevas significaciones de valores que se reflejan en su comportamiento frente a sus alumnos y la sociedad. Se destruyen las significaciones de los valores que sustentaban la orientación hacia el ser humano $y$, en contrapartida, no se observa de forma paralela la formación de otros referentes sustitutos, sino que se yuxtaponen aquellos impuestos por los modelos externos individualistas. Así, se vive en una ausencia de reglas colectivas que favorezcan un individualismo responsable. Y aquí entran los referentes éticos.

Dentro de estos referentes éticos estarían los valores universales, que según la opinión de algunos entrevistados nunca fueron universales, pues la historia de la humanidad nos enseña que ha habido sistemas de valores muy diversos en las diversas culturas. No obstante, nosotros los consideramos como universales en el sentido de que proporcionan un sentimiento de seguridad a cada sistema de valores en particular. Surgiendo aquí otro pensamiento, que durante mucho tiempo, la sociedad percibía solamente los puntos positivos de estos grandes relatos y, recientemente, en las últimas décadas algunos grupos han puesto su atención en los aspectos negativos, que paradójicamente se derivan de los positivos, inclinándose fuertemente en dichos aspectos negativos e influenciados por la corriente posmoderna, dan lugar al derrumbamiento de los metarrelatos. Con ello, observamos cómo determinadas corrientes acaban traicionándose a sí mismas, ya que en la búsqueda del reconocimiento de lo negativo, de la destrucción de los mitos, acaban construyendo otros mitos, los cuales no llevan al 
sentimiento de seguridad y tampoco proponen algo como referencia para que el ser humano invierta en sí mismo y en su formación.

En lo que se refiere a los valores, con la finalidad de rescatar la parte humana, uno de los valores que debería sustentar la universidad sería más que la búsqueda de la verdad, la búsqueda de la justicia, de la equidad, de la tolerancia. Los valores fundamentales, como la solidaridad intergeneracional y el cuidado de la vida, estarían siendo sustituidos por otra visión que considera primordialmente al individuo aislado en términos de sus posibilidades económicas. La universidad debería buscar la formación con base en valores de índole universal que coadyuvan a minimizar el valor cultural tan generalizado que reza que solamente a través de la obtención de bienes materiales, se puede lograr la felicidad.

La pérdida del humano se agudiza todavía más con la tecnología, la cual se convierte en una ideología que cierra los espacios para la discusión humanista. Al mismo tiempo, los cambios en la sociedad se han dado tan rápidamente que no permiten replantear los conceptos de los valores tradicionales, morales y éticos. Todo este escenario se ve reflejado en los programas de estudios de la universidad, en los que predomina la preocupación por la cantidad de personas tituladas, sobre la calidad de la formación. Del mismo modo, la universidad ya no proporciona a los alumnos la posibilidad de obtener sus propias conclusiones de los valores pregonados en cada momento histórico y, mucho menos, que ejerzan su libertad de pensamiento para elegir sus propios valores y aplicaciones. Todos estos fenómenos ratifican la falta de convicción y orientación que los alumnos tienen en lo que se refiere a sus vidas en general.

Al considerar si los valores se conforman y son percibidos nacional y regionalmente, algunos entrevistados señalan que persisten algunos valores que caracterizan al mexicano, tales como la falta de responsabilidad y compromiso personal para ejercer la propia libertad de pensar y tomar decisiones. Este hecho podría reflejar el proceso histórico, económico y político que la nación ha vivido durante siglos y en el que la población ha estado condicionada a una ideología autocrática, paternalista y reguladora. Tal vez de ahí se origina la idea de que la universidad deba seguir fomentando valores como el respeto, la disciplina y el trabajo, así como la transmisión de confianza al alumno a través de una postura ejemplar del profesor basada en el caudal de conocimientos que éste último maneja. Los profesores comentan que una cosa importante, es darle confianza al alumno y motivarlo en una cierta tarea.

Que el alumno vea a los profesores como seres humanos, quienes cometen errores, pero que cometen el menor número de errores posible en clase, en los conocimientos de la materia. Sienten que es una motivación para los alumnos empezar a 
ver al profesor, qué tanto domina el tema, además de la responsabilidad de cumplir con tareas. El valor primordial que permearía en la docencia sería la presencia de la sociedad orientando las decisiones e indicando cuál es la significación real de lo que se está haciendo. La transformación sería posible si el alumno fuera capaz de asumir la responsabilidad de la significación. Consecuentemente, el conocimiento sería transformador en el sentido de que liberaría a todos los que estamos atrapados en las reglas estrictas del positivismo.

Por otra parte, se constata la afirmación de que la universidad posee valores fundamentales que son: la libertad de cátedra, la libertad de expresión y de opiniones $\mathrm{y}$, al mismo tiempo, que ella ofrece a los alumnos la oportunidad de que se integren a la sociedad a través del servicio social y las prácticas profesionales. En medio de este torbellino de valores que orientan a la universidad, o que deberían orientarla, los profesores entrevistados expresan una preocupación general sobre la posibilidad de formar sujetos emancipados, pues, según sus opiniones, éstos están disminuyendo cada vez más.

Las razones que propician esto se resumen en que la universidad, frente a un proceso de globalización, está cediendo ante las presiones tecnologistas y productivistas presentes dentro de la sociedad. Y en este sentido los alumnos estarían perdiendo incluso la posibilidad de liberarse, pues absorben las tendencias de aprender técnicas y abandonan la posibilidad de desarrollar la facultad de pensar y buscar relaciones entre la teoría y la práctica que les permitirían tener una visión crítica; y al mismo tiempo, los alumnos sólo se insertan en el contexto de producir en términos de bienes materiales.

De esta forma, la propia universidad vive una gran contradicción: debe ser reguladora para cumplir con las demandas del mercado global al formar personas operativas; al mismo tiempo que debe rescatar los aspectos filosóficos de valores como la responsabilidad, la honestidad, la honradez, la libertad, la lealtad, la visión de vida... Como ejemplo de esta contradicción, se observa la imposibilidad de ejercer la libertad ante la necesidad de tomar un trabajo, aun cuando éste no permita a la persona desarrollarse ampliamente.

Interpretando emancipación desde el concepto de Freire, se observa que dentro del contexto universitario no es conocida y mucho menos entendida. Sin embargo, en las comunidades marginales, donde el nivel de escolaridad es mínimo, la búsqueda de la emancipación es un hecho que se observa en la práctica. En la universidad, la emancipación es una idea que incluso pierde sentido frente a un mundo que exige ser el mejor o el número uno, impidiendo que una gran masa se emancipe, 
prueba de ello, es la ausencia de reflexión y crítica que muestran alumnos de áreas dedicadas primordialmente al cuestionamiento social, cuyas observaciones son débiles $\mathrm{y}$ de poco alcance.

Hoy se percibe que los grupos que se articulan buscando, a su manera, una vía de emancipación, lo están haciendo a través de actitudes y hechos violentos, que en el fondo están respondiendo a una serie de violencias anteriormente sufridas por ellos, es decir, actos violentos de los que han sido víctimas y que no se han evidenciado por estar diluidos en la cotidianeidad. No obstante, que estas manifestaciones sean interpretadas como actos violentos, estando o no de acuerdo, se podría decir que son los inicios de movimiento o tendencia a organizarse para lograr la emancipación.

Por otra parte, existe la preocupación sobre la vinculación entre la universidad y la sociedad; sin embargo, los investigadores se encuentran inertes en cuanto a los canales que podrían establecer estos vínculos, viendo como única posibilidad para esto la mayor difusión y divulgación de sus trabajos. Las investigaciones se desarrollan dentro de líneas muy particulares, lo que dificulta la aproximación a las grandes demandas y desafíos generados por la sociedad. No obstante, se puede percibir que algunas áreas, como los estudios sobre el agua, tienen un campo muy amplio de aplicación y posibilidades de obtener sólidos resultados, debido a las repercusiones que tienen en todos los sectores de la sociedad.

La voluntad de repensar, buscar soluciones, encontrar respuestas que traigan seguridad, esperanza y utopías, está presente en la voz de los profesores investigadores, en sus miradas, en sus rostros, y en sus emociones. Ellos mismos se vuelven protagonistas del problema: por la soledad de su trabajo y el reconocimiento de la impotencia individual para generar un cambio colectivo y profundo en las estructuras de la institución, cada historia personal, como diría Habermas, se ve invadida por el mundo de la vida, por el sistema.

\section{FINALIZANDO}

Quizás, como educadores, y específicamente como investigadores, tenemos un arduo trabajo por realizar. Tenemos que pensar cómo vamos a trabajar para la construcción de un mundo más libre, responsable, ético, democrático, participativo, equitativo, fraterno y con valores que formen un ciudadano con buenos sentimientos, solidario, crítico y emancipado. Es necesario pensar al hombre en su totalidad, como ser que tiene la incansable tarea de humanizarse, completarse. 
Es necesario pensar al hombre como un proceso no concluido y para ello, urge que pensemos y que nos preguntemos constantemente sobre nuestras formas y objetivos al educar e investigar. Esto implica dar un giro hacia nosotros mismos, buscar nuestra construcción como seres individuales, para después irradiar la teoría en la práctica con base en ejemplos y acciones dentro del mundo cotidiano que sean coherentes e irrefutablemente testimoniales.

El profesor investigador tiene la tarea de re-construirse e innovar su docencia con una pedagogía permeada por didácticas llenas de práctica, que inviten a sus alumnos a afrontar la realidad, a prepararse para dar respuestas a sus miedos, a sus problemas individuales, pero sobre todo con estructura, creatividad y conocimientos para actuar en sus comunidades. Una vez superados nuestros propios miedos, inexperiencias, ignorancias, existe la necesidad de luchar con perseverancia, con insistencia para que sean creadas políticas educativas que consideren la infraestructura para la investigación, así como para la docencia, difusión y extensión de la cultura y de los conocimientos.

La lucha por políticas públicas orientadas a múltiples necesidades: a un piso salarial mínimo obligatorio a ser pago a los profesores de todos los estados de la nación y, en el caso de que algún estado no tenga la suficiente recaudación para cumplir con los compromisos sociales, pudiera, a través de una gestión transparente, recibir auxilio del gobierno federal; tener incentivo y estrategias determinadas por las universidades para la conformación de equipos multidisciplinarios para realizar las investigaciones; oportunidades de acceder a mayores informaciones bibliográficas, así como a la tecnología y, de igual forma, tener oportunidades para capacitarse para saber usarlas, emplearlas no solo en las investigaciones sino en los salones de clase; establecimientos de redes nacionales e internacionales, para buscar las experiencias ya existentes y cómo funcionan; participación de congresos internacionales de transcendencia académica; apoyo para la publicación de los resultados de sus investigaciones y, principalmente la posibilidad de llevar las descubiertas a la aplicabilidad.

También se necesita crear espacios en donde las voces de todos los actores sean escuchadas y se tornen un único discurso con el objetivo de generar el bienestar de todos. Espacios estos que en muchos países se conforman a través de sindicatos, asambleas, movimientos...sin embargo, bien organizados, articulados bajo una orientación de estrategias inteligentes, donde la palabra y el argumento posean su carga de poder, pues para ello se necesita de información, conocimientos, pruebas, documentaciones, debates con seres pensantes, respetados por su labor honesto y de aportaciones a la nación. Caso contrario, se corre el risco, que hoy por hoy impera, 
que es el no seguimiento y cumplimiento de las pocas posibles políticas educativas existentes, permeados por la suma de todos los matices del descaso y de la corrupción.

En el caso de México, donde está ubicada la Universidad en que se realizó el estudio, las decisiones de las políticas educativas se quedan en las manos de quienes ni si quiera poseen la noción de la labor de la educación y mucho menos poseen la formación necesaria, puesto que la educación es política y no pedagógica. Los ejemplos que tenemos en varios micros espacios de instituciones de educación superior son rectorías y secretarias ocupadas por personas sin la calificación, experiencias y conocimiento de lo que involucra su labor, sus funciones.

Incluso, como el maratón para cursar posgrados fue detonado como un motor de consumo: la mayoría quien ocupa cargos de toma de decisión se inscriben en maestrías y doctorados sin calificación y reconocimiento en el ranking de las evaluaciones de calidad de los posgrados. La obsesión por ser doctores a cualquier precio, no importa, lo que importa es ser llamado de doctor. Saber de gestión, saber colocar instrumentos de medición que garanticen que no alcanzaran los estímulos eso es ser un buen gestor. Así, en esta re significación de valores y ética, el poder no rebuzna porque no encuentra el tono; sin embargo, la comunidad académica tiene que subordinarse, bajo el silencioso terrorismo de la punición.

La realidad desvela la más perversa de las tecnologías del yo. Por tanto, hablar de la docencia universitaria, de la investigación y extensión del conocimiento es un tema que ultrapasa los salones de clases: va desde una re-construcción de ser humano, del propio profesor; de su filosofía de vida en creer en la esperanza; en su valentía y carácter de no silenciar frente tantos abusos y humillaciones; en su ciudadanía política y militar caso necesite diseñar las estrategias; en su participación activa en los movimientos de justicia, siempre guardando las evidencias para una posible posterior defensa de sus derechos y de su clase. Así como toda la educación transita entre el equilibrio de la teoría y de la práctica, hablar acerca de la universidad y de sus funciones y del profesor es incursionar por el equilibrio entre los discursos y las vivencias. Hoy, la tarea es grande para volverse un profesor transformador, innovador, no fragmentado, pero mientras existan las utopías, se vale luchar.

\section{REFERENCIAS}

ADORNO, W. T. Dialéctica Negativa. Madrid: Taurus, 1989.

ADORNO, W. T. Consignas. Argentina: Amorrortus, 1993. 
ADORNO, T.; HORKHEIMER, M. Dialéctica de la Ilustración. Madrid: Trotta, 1994.

DEWEY, J. The Relations of Theory and Practice. Southern Illinois: University Press, 1977.

FREIRE, P. Pedagogía del Oprimido. México: Siglo XXI, 1974.

GIROUX, H. Los Profesores como intelectuales hacia una pedagogía crítica del aprendizaje. Barcelona: Ediciones Paidós, 1988.

GIROUX, H . "O Pós - modernismo e o Discurso da Crítica Educacional”. In: SILVA, T.T. (Org.). Teoria Educacional Crítica em Tempos Pós - modernos. Porto Alegre: Artes Médicas, 1993.

GRAMSCI, A. La Formación de los intelectuales. México: Grijaldo, 1967.

GRAMSCI, A. Los intelectuales y la organización de la cultura. México: Juan Pablos Editor, 1997.

HABERMAS, J. Historia y Crítica de la opinión pública. Barcelona: Ediciones Gustavo Gili/Masd Media, 1981.

HABERMAS, J. Conhecimento e interesse. Rio de Janeiro: Zahar, 1982.

HABERMAS, J. EI Discurso Filosófico de la Modernidad. Madrid: Taurus, 1989.

HABERMAS, J. Ensayos Políticos. Barcelona: Ediciones Península, 1988.

HELLER, A.; FEHÉR, F. El Péndulo de la Modernidad. Barcelona: Ediciones Península, 1994.

HELLER, A.; FEHÉR, F. Políticas de la Postmodernidad - Ensayos de Crítica Cultural. Barcelona: Ediciones Península - Historia, Ciencia, Sociedad, 1998.

HELLER, A. O Cotidiano e a História. São Paulo: Paz e Terra, 1982a.

HELleR, A. Para Mudar a Vida. São Paulo: Brasiliense, 1982 b.

HELLER, A. "Un Siglo que toca a su fin”. Revista Internacional Letras, México, n. 49, p. 9-12, 1997.

LIPOVETSKY, G. La Era del Vacío - Ensayos sobre el individualismo contemporáneo. Barcelona: Anagrama, 1986. 
PUIGGRÓS, A. Imaginación y crisis en la educación latinoamericana. México: Alianza Editorial, 1990.

SANTOS, B. Introdução a uma ciencia pós-moderna. Rio de Janeiro: Graal, 1989.

SANTOS, B. Um Discurso sobre as Ciências. Portugal: Edições Afrontamento, 1997.

SANTOS, B. Conhecimento Prudente para uma Vida Decente. "Um Discurso sobre as Ciências". São Paulo: Cortez, 2004.

SANTOS, B. A gramática do tempo. São Paulo: Cortez, 2008.

Recebido em: 09 de agosto de 2016 Aceito em: 29 de novembro de 2016

Endereço para correspondência: CLLE Independencia MZ 3 LT 20, INT C 1007 Geovillas de la Independencia, Toluca Mex C.P.50228, México; arisan3@gmail.com 
